\title{
Quantum cascade laser sensors for online gas chromatography
}

\author{
Sheng Wu, Andrei Deev, Yongchun Tang \\ PEER Institute \\ 738 Arrow Grand Circle, Covina, CA 91722 \\ California Institute of Technology \\ 139-74 Caltech, Pasadena, CA 91125
}

\begin{abstract}
We show that QC laser could improve capillary Gas Chromatography Infrared spectroscopy resolution significantly, i.e. both Doppler limited and Doppler free resolution could be achieved. To achieve these goals, we report our latest efforts in characterizing the tuning and noise properties of Quantum Cascade (QC) lasers; novel schemes on modulation to gain largest tuning range as well as on stabilizing and locking the QC lasers are proposed, and results presented.
\end{abstract}

KEY WORD LIST

Current tuning, Mid Infrared, Whispering Gallery Mode, Quantum Cascade Laser, Saturated absorption, hollow waveguide

\section{INTRODUCTION}

\section{Reviving Infrared detector for Gas Chromatography with Quantum Cascade Lasers}

Thirty five years ago, when the leading Gas Chromatography instrument company Agilent, then Hewllet-Packard, introduced capillary GC and bench top detector MSD (Mass Spectrometry Detector), they also introduced another detector that could uniquely identify the chemical elute peaks and it was based on Infrared detection (IRD) using FTIR as the spectroscopic identification tool. Over the decades, HP/Agilent sold hundreds of thousands of GC-MSDs, but the GC-IRDs are in the lower 100s. The reason behind this sales/market disparity is the poor sensitivity of IRD. Infrared detection is not necessarily a method associated with poor sensitivity, given the fact that molecules often have very strong absorption in the Mid-IR. The bottleneck was that for blackbody sources used in FTIR, the light sources could not be efficiently coupled into capillary IR light waveguide, resulting in very short optical path, and also enlarged bore size and accompanying dilution by makeup gases further degrade the performance of IRD[1]. With the advent and advances of Quantum Cascade lasers, we demonstrated that very high coupling efficiency for QC lasers into IR hollow waveguide (HWG) could be realized[2]. This effectively increased the pathlength of optical absorption to meters from inches, and with no sacrifice in markup gas dilution because the possibility to use small bore HWG. The fast scanning and high spectral purity capabilities of QC laser further promise to improve the performance of GC-IRD close to that of GC-MSD. Yet, as QC lasers continue to make strides forward, e.g. Continuous Wave (CW) high power room temperature operation [3, 4] and wide scanning[5] capabilities are rolling out of laboratories, the performance of GC-IRD could be further improved. Here, we show that our efforts to demonstrate wide and fast scanning of CW QC DFB lasers; locking/stabilizing CW QC DFB laser frequency to realize Doppler limit and Doppler free spectral resolution inside HWG. The Doppler limit resolution will improve the GC-IRD based on QCL to the same level as regular tunable diode laser spectroscopy, and over one order of magnitude better than the best bench top FTIR; while the Doppler free resolution will further put QCL powered GCIRD to a resolution never achieved before.

Quantum Sensing and Nanophotonic Devices VIII, edited by Manijeh Razeghi, Rengarajan Sudharsanan, Gail J. Brown, Proc. of SPIE Vol. 7945, 794506 - @ 2011 SPIE · CCC code: 0277-786X/11/\$18 · doi: 10.1117/12.871398 


\section{Modulating and modifying QC Lasers for chemical sensing}

A major challenge for QC laser application in chemical sensing spectroscopy is how to tune the laser frequency or wavelength over a range as wide as possible and at the fastest speed possible.

Distributed Feedback (DFB) QC lasers operate on inherently single frequency, but their tuning range is limited. We show that Continuous Wave (CW) QC DFB lasers, due to its high thermal load and relatively wide working temperature range, could tune over $10 \mathrm{~cm}^{-1}$ in a single current ramp pulse as short as $100 \mu \mathrm{sec}$, thus giving more capability for this kind of robust QC lasers for chemical sensing. In this paper, we tested the tuning range by using this laser to measure the $\mathrm{CO}_{2}$ dissolved in water. Due to strong absorption in the MIR by water, direct spectroscopic measurement of $\mathrm{CO}_{2}$ in water has been limited to optical path lengths of only dozens of microns, and measurable concentration resolution of only $200 \mathrm{mg} / \mathrm{L}$. The high power and brightness of Quantum Cascade lasers could penetrate much deeper optical depths[6-8], i.e. 100s microns, and providing much higher averaging speed to achieve higher concentration resolution. We demonstrate that we could penetrate as deep as 800 micron of water with our tunable EC QC laser built upon the amplifier above.

Even with $10 \mathrm{~cm}^{-1}$ tuning range, the DFB QC laser's tuning range is still well below the full gain bandwidth of QC lasers --- usually runs from $60 \mathrm{~cm}^{-1}$ to over $400 \mathrm{~cm}^{-1}$. External cavity with grating could tuning the full range as to over $400 \mathrm{~cm}^{-1}$, but compromised on tuning speed.

\section{Linewidths for hetero-structure epi lasers}

Near-IR DFB and External Cavity diode lasers

DFB diode lasers are the most mature and stable single frequency laser sources, especially in the Near-IR (NIR) telecom band. However, the inherent high frequency noise in free-running (only constant current and temperature and without external frequency locking or stabilization) DFB NIR lasers broadens their Instantaneous Linewidths (ILW, $<100 \mu \mathrm{sec}$ average) to over several MHz. This broadened ILW is actually a merit for DFB lasers working in the telecom, because the broadened ILW will suppress noises generated in the long fiber loop by stimulated Raman scattering and other nonlinear effects.

The past experiments in the NIR with External Cavity (EC) diode laser with a grating as frequency selected feedback suggest that their ILW is much narrower, and $\sim 500 \mathrm{KHz}$ was demonstrated[9] by high precision spectroscopy or high Q cavities, and in telecom more often by Delayed Self-Homodyne RF analysis[10].

The frequency drift, i.e. over msec time scale, of a free running single frequency NIR diode laser under constant temperature $\left( \pm 0.1^{\circ} \mathrm{C}\right)$ and bias current is much larger. This large drift is a result of the varying background temperature causing the internal temperature to drift, and also drift of the current in the laser driver.

\section{Linewidths of DFB and EC Quantum Cascade Lasers}

Free running DFB QCLs are expected to have much narrower ILW than those of conventional Near-IR (NIR) diode lasers operating at the same power. This is because the photon energies are smaller, the cavities are longer $(2 \mathrm{~mm}$ versus $0.2 \mathrm{~mm}$ ) and the linewidth enhancement parameter alpha, or $\alpha$, is expected to be near zero. The first two of these factors reduce the Schawlow-Townes linewidth[11], while the large alpha parameter of NIR diode lasers typically increases the free-running ILW by factors of 10-40 above the Schawlow-Townes limit. It is demonstrated that with low noise drivers, free running DFB QCLs could achieve a linewidth of $150 \mathrm{kHz}$, and the SchawlowTownes linewidth could be much less than $100 \mathrm{kHz}$ for many DFB QCLs[12, 13]. But, detailed spectroscopy evidence was not demonstrated. Frequency stabilization of QCLs further decreases ILW and long term drift to KHz level and facilitates sub-Doppler spectroscopy, but the stabilization setup which involve external cavity or external saturated absorption is quite complicated[13]. 
So far, a linewidth of 3.99MHz has been demonstrated for a Mid-IR EC QCL[14], and further stabilization on the EC QCL should prove much finer linewidth like ECL diode lasers stabilized in the NIR. Similar ILW for DFB QC Lasers were reported, and simple external locking has only been able to reduce long term drift and ILW is still several $\mathrm{MHz}[15]$. There is hope that with improved low noise driver, the DFB QCL and EC QCL could achieve linewidths below a couple of $100 \mathrm{kHz}$ without complicated external locking. Here, we investigate the ILW for EC QCL based on the amplifier design above and also for the commercial DFB QCL.

\section{How to measure and characterize the ILW of QC lasers and applications}

So far, the ILW has to be verified by Saturated Absorption (SA) or cavities with ultrahigh Q in the MIR for EC QCLs.

The ultra narrow Doppler free linewidth of stable molecules is a good benchmark gauge for the ILW of QC lasers, be them DFB or EC QC lasers. We could conduct them easily in a compact setup, e.g. Hollow Waveguide (HW). Compared with traditional free space overlapped counter propagating SA setup, the tight overlap is guaranteed in a hollow waveguide as long as the laser could be efficiently coupled into the waveguide. We demonstrated that the coupling efficiency is over 50\% for semiconductor epi lasers, e.g. QC lasers, due to QC laser's high brightness[2]. The transmission loss of $\mathrm{HW}$ is also quite low, only about $1-2 \mathrm{~dB} /$ meter, making it an ideal platform for SA spectroscopy.

To measure the $100 \mathrm{kHz}$ linewidth of a DFB or EC QCLs with a high Q cavity at $5 \mu \mathrm{m}\left(6 \times 10^{13} \mathrm{~Hz}\right)$, the Q should be over $6 \times 10^{8}$, and a whispering gallery mode cavity based on Fluorite crystal discs should be able to realize such Q easily.

\section{Applications of Saturated Absorption and WGM in the Mid-IR}

The application of Doppler free ultra-high resolution SA spectroscopy in the MIR had been forecasted ever since its first demonstration[16]. Basically, the Doppler limited spectroscopy has a frequency over resolution of $\sim 6 \times 10^{5}$, e.g. at $2,000 \mathrm{~cm}^{-1}$ and Doppler limited resolution of $0.003 \mathrm{~cm}^{-1}$, while the Doppler free SA's spectroscopic resolution, i.e. $100 \mathrm{kHz}$ or $3 \times 10^{-6} \mathrm{~cm}^{-1}$, will lead to nearly 3 orders magnitude increase of information and enable the differentiation of many chemical species previously impossible, e.g. nuclei isomers[17], magnetic hyperfine structures. The major obstacles for SA have been the lack of tunable lasers in the MIR, where the molecules have strong enough absorption to create SA, and the complicated platform of SA spectroscopy. Now, with widely tunable QC and intersubband MIR lasers, and the hollow waveguide, SA could be easily used for real world applications, e.g. the identification of isotopomers of heavy molecules, e.g. UF6, SF6[18], ethane[19], propane.

It has generated lots of interests in the past decade as Whispering Gallery Mode resonators can have ultra-high Q factors over a wide wavelength range in a tiny cavity, which usually could not be realized with conventional cavities with dielectric coatings[20,21]. The paths of the WGMs lie very close to the surface of the resonator with a portion of the electromagnetic (EM) wave traveling outside, i.e. evanescent wave. Losses due to absorption or scattering of the evanescent wave outside the resonator will change the $\mathrm{Q}$ factor of the resonator. Several groups demonstrated that WGM resonators could be used for absorption sensing in gases and liquids, detection of refractive index changes around the resonator and single molecules on its surface[22, 23]. All chemical sensing studies performed until now with WGM used near-IR and visible continuous wave (CW) single frequency lasers because of the wide availability of such lasers and detectors. However, mid-Infrared (MIR) and far-Infrared (FIR) regions of the spectrum are much more important for chemical sensing because the fundamental bands of vibrations lying in these regions are 1 3 orders of magnitude stronger than their near-infrared overtones. However, there are some difficulties in realizing MIR/FIR coupled WGMs., i.e. there are very few choices of single mode fibers in the MIR/FIR, and the most common fused silica material is not transparent in that range, and finally CW QCL sources are still far from as popular as telecom CW diode lasers, but the latest improvements in QCLs bode well for their applications. 
Because of the high brightness of QCLs, we recently demonstrated that the radiation of a QC laser could be efficiently coupled into a $300 \mu \mathrm{m}$ diameter hollow waveguide and used as a microliter gas sensor in gas chromatography. We also demonstrate that pulsed QCLs, in spite of their relatively large linewidth, could be efficiently coupled into WGM cavities.

In this paper, we demonstrate, to the best of our knowledge, the first SA spectroscopy of molecules in a hollow waveguide with unprecedented overlap and saturated intensity. Also, we demonstrate, the first observation of WGM spectra with Q well over 1 million in the MIR excited with a CW laser.

\section{EXPERIMENT}

Fast and Wide Frequency Tuning with Low duty cycle current ramp pulses

The schematic for fast and wide frequency tuning for DFB QC lasers is given in figure 1. The laser output is collimated and coupled into a WGM cavity with $0.47 \mathrm{~cm}-1$ Free-Spectra Range (FSR), and by counting the period in each current ramping cycle, we could calculate the frequency tuning range as well as mode purity or mode hopping.

\section{WGM observation with DFB QCL}

The setup is similar to the experiment where we demonstrated the pulsed QCL coupling into WGM reported last year. The detector has a bandwidth of $20 \mathrm{MHz}$, and the scan rate of the DFB laser has to be slow enough to let the detector resolve the sharp features of the WGM spectra. But at slow scanning rate, the jitter of the DFB QC laser will become the major limiting factor. The free running DFB QC lasers have been demonstrated to have an estimated bandwidth of over $10 \mathrm{MHz}$ at $1 \mu \mathrm{sec}$ scale. Therefore, the observable $\mathrm{Q}$ at $2,280 \mathrm{~cm}^{-1}$ will be limited to $8 \times 10^{6}$.

We carefully adjusted the angle of incidence (AOI) of the QCL beam at the hypotenuse face so that only the lowest order WG modes, i.e. $q=1$, are excited. We achieved near single mode excitation for $q=1 \mathrm{WG}$ modes when we adjust the AOI of QCL at the hypotenuse to be slightly above the critical angle for total internal reflection to happen at the $\mathrm{ZnSe}$ and $\mathrm{CaF}_{2}$ interface, which is $35.3^{\circ}$ at $4.5 \mu \mathrm{m}$ wavelength. As AOI getting closer to this critical angle, the WGM structure starts to simplify.

We obtained $\mathrm{CaF}_{2}$ discs by OEwaves using procedures for fabricating high $\mathrm{Q} \mathrm{CaF}_{2}$ WGMs[24], we also fabricated $\mathrm{CaF}_{2}$ and $\mathrm{BaF}_{2}$ discs with different morphology on the outskirt. Results of these high Q WGM resonators will be obtained in the coming weeks.

\section{Saturated Absorption in Hollow Waveguide with EC and DFB QCL}

We coupled laser output from our EC and DFB QCL into the $300 \mu \mathrm{m}$ ID HW, and achieved $>50 \%$ coupling and over $40 \%$ output after 0.5 meter HW length. The laser output from the other side of the HW is back reflected with a concave gold coated reflector, matching the Numeric aperture of the HW. The back-reflected signal is sampled by a $\mathrm{ZnS}$ beam splitter and detected by a MCT detector. The $\mathrm{ZnS}$ beam splitter has a Fresnel reflectivity of $>20 \%$ at each surface at $45^{\circ}$ Angle of incidence, and therefore a total of $4 \%$ each face of power is reflected into the detector after double passing the HW.

\section{RESULTS}

Figure series 2 demonstrate the current tuning capability when we use the current ramping pulse scheme as discussed above and described in figure 1 . With $2 \%$ duty cycle pulse and $100 \mu \mathrm{sec}$ pulse width $(200 \mathrm{~Hz})$, TEC reached $-45^{\circ} \mathrm{C}$ versus $-34^{\circ} \mathrm{C}$ with full duty cycle triangle waveforms. The chirp maximum rate is estimated to be $0.2 \mathrm{~cm}^{-1} / \mu \mathrm{sec}$, or $6 \mathrm{MHz} / \mathrm{nsec}$, and according to reference[29], the rapid passage effect should be negligible in most spectroscopic applications. The chirp rate could also be minimized by reducing current ramp rate after passing the maximum current value, i.e. $0.5 \mathrm{~A}$ in this particular QCL device. As TEC temperature change from $+28^{\circ} \mathrm{C}$ to $-45^{\circ} \mathrm{C}$, the wavelength scan range changed from 4 peaks to 6 peaks of ${ }^{13} \mathrm{CO} 2$, corresponding to $\sim 9 \mathrm{~cm}^{-1}$ to $15 \mathrm{~cm}^{-1}$. Also, the 
power goes up 3 times, while the effective current tuning range changed increased $>50 \%$. If the laser is operated with traditional $100 \%$ duty cycle triangle pulses with a baseline current at threshold, then the tuning range will be further limited to 2 peaks of ${ }^{13} \mathrm{CO} 2$, i.e. $\sim 5 \mathrm{~cm}^{-1}$, when operating at $15^{\circ} \mathrm{C}$. Therefore, by operating the laser at low TEC temperature of $-65^{\circ} \mathrm{C}$ versus $+15^{\circ} \mathrm{C}$, we gain an extra tuning range $\sim 16 \mathrm{~cm}^{-1}$, and using low duty cycle fast ramping, we gain an extra $3 \mathrm{~cm}^{-1}$ tuning range. This gives over $20 \mathrm{~cm}-1$ tuning range in less than $100 \mu \mathrm{sec}$ ramp. Such wide and fast tuning capability could theoretically be maximized with optimized parameters for the devices, and should reach over $1.5 \%$ of the center frequency.

Figure series 3 show the WGM spectra. A maximum WGM coupling induced loss of $10 \%$ is observed when optimized. The period spacing between the WGM spectra is measured to be $\sim 0.45 \mathrm{~cm}^{-1}$, which is calibrated with an air spaced Ge etalon with a Free Spectral Range of $0.075 \mathrm{~cm}^{-1}$. This value is consistent with the size of the $\mathrm{CaF}_{2}$ ball lens, i.e. $5 \mathrm{~mm}$ diameter. In fact, we noticed that the WGM modes for this $5 \mathrm{~mm} \mathrm{CaF}_{2}$ ball are much simpler at $4.46 \mu \mathrm{m}$, when compared to the case at $1.55 \mu \mathrm{m}$, i.e. with the same setup at the critical AOI for $1.55 \mu \mathrm{m}$ as shown in the insert of figure 2. We attribute this reduction in the number of WGM dips to the facts that we are using a $4.46 \mu \mathrm{m}$ laser, instead of a $1.5 \mu \mathrm{m}$ laser. The longer wavelength laser reduces the equator's WGMs to almost single $q=1$ operation under the critical AOI coupling. The measured Q is over $2 \times 10^{6}$ in the MIR of $2,280 \mathrm{~cm}^{-1}$.

Figure series 4 show the Doppler limited spectral resolution when the HWG is coupled upstream to GC capillary and downstream with a vacuum system. Roughly $140 \mathrm{MHz}$ linewidth is demonstrated when the pressure is reduced to 40Torr, i.e. when the pressure broadening is reduced to below that of Doppler limit at $2,260 \mathrm{~cm}^{-1}$. The broadened line width after average is a result of the noisy QC driver which will be replaced with a low noise driver. All spectra figures were taken with an inlet flow rate of $1 \mathrm{cc} /$ minute and pressure sensors read $40 \pm 0.5$ Torr on both the inlet and outlet.

\section{DISCUSSIONS}

\section{Thermal tuning capability of DFB QC lasers versus DFB Diode lasers}

Here, we calculate the change of resonance wavelength and frequency for DFB laserswith every Kelvin change in core temperature. The equations go out like this,

$$
\begin{gathered}
\lambda_{\mathrm{DFB}}=2 n \Lambda / m ; \quad \text { or } \quad d \lambda_{\mathrm{DFB}} / \mathrm{dT}=(2 \Lambda / m) d n / d T ; \\
O r, \quad d \lambda_{\mathrm{DFB}} / \mathrm{dT}=\left(\lambda_{\mathrm{DFB}} / n\right) d n / d T
\end{gathered}
$$

Where $\lambda_{\text {DFB }}$ is the resonance wavelength, $\boldsymbol{n}$ is the refractive index, $\boldsymbol{\Lambda}$ is the pitch of the grating and $\boldsymbol{m}$ is the order of the grating $\mathrm{T}$ is the grating waveguide temperature which is just above the active region (AR). $\boldsymbol{d n} / \boldsymbol{d} \boldsymbol{T}$ is a material constant and varies little as long as InP composition does not change much from diode laser to QC laser, and so let's define a quasi constant $\boldsymbol{\beta}=\boldsymbol{d n} / \boldsymbol{d T} / \boldsymbol{n}$, and we get:

$$
\begin{gathered}
d v_{\mathrm{DFB}} / d T=-\left(c / \lambda_{\mathrm{DFB}}{ }^{2}\right) d \lambda_{\mathrm{DFB}} / d T=-\left(c / \lambda_{\mathrm{DFB}}\right) \beta=v_{\mathrm{DFB}} \beta \\
\text { and }, \quad d \lambda_{\mathrm{DFB}} / \mathrm{dT}=\lambda_{\mathrm{DFB}} \beta
\end{gathered}
$$

The latest result on a high temperature (423K) operation DFB QCL centered at $9 \mu \mathrm{m}\left(1,100 \mathrm{~cm}^{-1}\right)$ gives a $\beta$ value of $7.9 \times 10^{-5} / \mathrm{K}$, and a thermal power load tuning rate of $1 \times 10^{-3} / \mathrm{W}$ for epi-up mounting, and $0.52 \times 10^{-3} / \mathrm{W}$ for epi-down mounting[25]. 
Telecom DFB diode lasers have a typical tuning rate of $0.08 \sim 0.12 \mathrm{~nm} / \mathrm{K}$ at $1.5 \mu \mathrm{m}$ or $10 \sim 15 \mathrm{GHz} / \mathrm{K}$, and $0.06 \sim 0.08 \mathrm{~nm} / \mathrm{K}$ or $18 \sim 24 \mathrm{GHz} / \mathrm{K}$ at $1 \mu \mathrm{m}[26]$--- which are also consistent with the $\beta$ value above for QC DFB lasers and also conclusions below.

So, we could draw the following conclusions for thermal tuning with DFB QC or diode lasers

- For the DFB lasers, regardless of QC or telecom diode, the temperature tuning frequency and wavelength rates both are proportional to the center wavelength or frequency. Therefore, for the same every Kelvin change in core temperature, QC laser in the mid-IR, e.g. $\lambda=4.5 \mu \mathrm{m}$, tunings 3 times as fast as in wavelength, but $1 / 3$ as slow as in frequency compared to diode lasers, e.g. $\lambda=1.5 \mu \mathrm{m}$.

- The tuning rates are not related to grating order or pitch.

- If we could change the AR temperature over $200 \mathrm{~K}$, e.g. through electric heating while maintaining the submount at low temperature, e.g. $-50^{\circ} \mathrm{C}$ or $223 \mathrm{~K}$, and assuming the maximum temperature for the active region is $430 \mathrm{~K}$, then we should have over $30 \mathrm{~cm}^{-1}(80 \mathrm{~nm})$ tuning range at $2,000 \mathrm{~cm}^{-1}$ center frequency $(5 \mu \mathrm{m}$ wavelength); or over $15 \mathrm{~cm}^{-1}(160 \mathrm{~nm})$ tuning range at $1,000 \mathrm{~cm}^{-1}$ center frequency $(10 \mu \mathrm{m}$ wavelength).

\section{Current tuning capability of DFB lasers through thermal heating effect only}

The compliance voltage for QC lasers $(7 \mathrm{v} \sim 17 \mathrm{v})$ is about $5 \sim 10$ times larger than diode lasers (1.5V typical), and efficiency $(<10 \%)$ is negligible when compared to diode lasers $(>35 \%)$, therefore the thermal power deposited on the QC lasers is about 10 times larger than diode lasers for the same current level or density.

The threshold current and current tuning dynamic range is also about 10 to 100 times higher for QC lasers, however, due to the fact that the width and length for QC lasers are much larger than telecom diode lasers (typical numbers are $10 \mu \mathrm{m}$ versus $2 \mu \mathrm{m}$ in width, and $3 \mathrm{~mm}$ versus $<1 \mathrm{~mm}$ in length), the current density for threshold current and current tuning dynamic range is only marginally higher for QC lasers compared to telecom diode lasers.

With BH structure, the thermal conductivity for QC lasers is similar to diode lasers, and so the thermal resistance is reduced, and typical thermal resistance of $10 \sim 20 \mathrm{~K} / \mathrm{W}$ is available for epi-up mounting and $10 \mathrm{k} / \mathrm{W}$ or less for epidown mounting[27]. Whereas, $\mathrm{BH}$ diode lasers have a thermal resistance of $100 \mathrm{k} / \mathrm{W}$ due to its smaller footprint[26].

If the thermal resistance is $\sim 100 \mathrm{~K} / \mathrm{W}$ for diode laser and $<10 \sim 20 \mathrm{~K} / \mathrm{W}$ for QC lasers, for the same every $m A$ current change, the temperature change is about the same for diode and QC DFB lasers. In order to thermally heat the AR over $200 \mathrm{~K}$, we need $20 \mathrm{~W}$ to $10 \mathrm{~W}$ power which means over $1 \mathrm{~A}$ current is needed. This might be over the maximum current limit already, and therefore we need to increase the thermal resistance. One way to increase thermal resistance is to increase the stripe width of the AR, and also use less gold deposition for ridge structures, and use epi-up mounting instead of epi-down mounting.

For telecom diodes, the maximum current is usually around $100 \mathrm{~mA}$, and therefore, the tuning range is limited to $150 \mathrm{GHz}$, or $5 \mathrm{~cm}^{-1}$, corresponding to an AR temperature increase of $10 \mathrm{~K}$; and for VCSEL, the Rth (thermal resistance) is $>1,000 \mathrm{~K} / \mathrm{W}$, and the heating effect is much stronger. QC laser will have a thermal tuning effect falling between the VCSEL an telecom diode, because of the total power is higher due to high working voltage, while its Rth is actually the smallest.

\section{Thermal tuning response dynamics and Low duty cycle wide range tuning}

The epi-down versus epi-up mounting will change the total temperature gradient from the AR core under static conditions, i.e. the current is held at constant; but during fast current ramp heating process, the gradient could be a lot higher than the static temperature gradient under a constant thermal load. 
If the QC laser has a low average power load, then the Active Region (AR) temperature will stay close to the lowest temperature possible by the TEC controlled submount. The maximum current will be decided by the maximum current density, as well as maximum AR temperature. It is possible to tune the thermal resistance, e.g. using larger AR width which increases the thermal resistance, or epi-down instead of epi-up to increase the thermal resistance so that the device's maximum current will be decided by the maximum AR temperature rise. Then, it is possible to apply a low duty cycle pulse to the QC laser, maintaining the lowest possible initial or zero current AR temperature.

An example pulse sequence could have a repetition rate of $1 \mathrm{kHz}$, and the pulse will first maintain zero current for $900 \mu \mathrm{sec}$, and let the AR temperature return to the submount temperature; then the pulse go from zero current to threshold in no time, i.e. $<0.5 \mu \mathrm{sec}$, and the AR temperature is close to submount temperature; and then ramp to maximum current allowed by the maximum AR temperature in about $100 \mu \mathrm{sec}$, then return to zero current for a repeating cycle. In this cycle, the average injected thermal power is only a fraction, $100 \%$ or less, of the full duty cycle ramp pulses.

\section{Thermal tuning coupled with multisection venier frequency tuning}

The epi-down versus epi-up mounting will change the total temperature gradient from the AR core under static conditions, i.e. the current is held at constant; but during fast current ramp heating process, the gradient could be a lot higher than the static temperature gradient under a constant thermal load.

\section{Saturated absorption inside hollow waveguide}

The currently demonstrated SA linewidth of $10 \mathrm{MHz}$ is mainly limited by the laser stability. If a stabilized laser, i.e. driven with low noise drive, the laser linewidth should be close to $100 \mathrm{kHz}$. Then, we could realized much higher resolution with SA in the HW.

The linewidth of the Saturated Absorption for stable molecules, e.g. $\mathrm{CO}_{2}$, is determined by several factors in the waveguide. The first is the transit time of the molecules inside the optical field due to the relatively small diameter of the HW. The transit time induced linewidth is calculated to be roughly following this equation,

$\mathrm{D}_{\mathrm{wall}} * \Delta v_{\mathrm{tr}} \sim 100 \mathrm{kHz} * \mathrm{~mm}$,

where $\mathrm{D}_{\text {wall }}$ is the dimension of the hollow waveguide, i.e. $350 \mu \mathrm{m}$, and $\Delta v_{\text {tr }}$ is the transit time limited linewidth will be $300 \mathrm{kHz}$. At a pressure of $\sim 0.05$ Torr, the pressure broadening $\left(\sim 4 \mathrm{MHz} /\right.$ Torr for $\left.\mathrm{CO}_{2}\right)$ will be $200 \mathrm{kHz}$--- less than transit time broadening. This means that the hollow waveguide could be coupled with GC and vacuum interfaced to provide ultra-high resolution spectroscopy analysis, if column switch is used to trap individual chemical peaks inside a long HW for analysis.

\section{WGM spectra in $\mathrm{CaF}_{2}$ ball/disc}

Since the energy loss in the ball is dominated by scattering loss, and Q is therefore proportional to the cube of the wavelength, as given in equation[28]:

$Q \approx \frac{3 \lambda^{3} a}{8 n \pi^{2} \bar{B}^{2} \sigma^{2}}$

where $\lambda$ is the wavelength, $a$ is the radius of the WGM cavity, $n$ is the refraction index of the resonator material, B is the correlation length, and $\sigma$ is the roughness. For the same $\mathrm{CaF}_{2}$ ball, we expect to observe $\sim 23$ times higher $\mathrm{Q}$ at $2,280 \mathrm{~cm}^{-1}$ than at $6,450 \mathrm{~cm}^{-1}$. Instead, we only observed roughly 4 times higher $\mathrm{Q}$. The missing Q could be obscured by the limited resolution of our laser, as shown in figure $3 \mathrm{f}$, we will use a Newfocus LB1005 fast servo-controller to to stabilize the laser to the sharp WGM fringes. Two WGM cavities could be used, one will stabilize the QC laser and 
scan the laser frequency by applying a PZT voltage to the cavity, and the $2^{\text {nd }}$ WGM cavity will monitor the linewidth of the stabilized QC laser.

As demonstrated with fluorite crystalline WGM cavities in the near IR, a $\mathrm{CaF}_{2}$ WGM resonator could potentially achieve values over $10^{9}$ for the $\mathrm{Q}$ factor at $1.5 \mu \mathrm{m}$ after careful polishing and annealing. Because of the longer wavelengths in the MIR and FIR, the main Q limiting factor will no longer be the surface scattering loss but the material absorption. This lower requirement on surface quality will make WGM sensors more robust in the MIR/FIR, i.e. they would be less sensitive to dust and environmental changes. $\mathrm{CaF}_{2}$ already starts to have higher absorption at the wavelengths over $4 \mu \mathrm{m}$ and limit its $\mathrm{Q}$ at $4.5 \mu \mathrm{m}$ to below $10^{9}$, while $\mathrm{BaF}_{2}$ could be a better candidate if we need to use longer IR wavelengths or get even higher $\mathrm{Q}$ factors at $4.5 \mu \mathrm{m}$.

Although fluorite crystalline WGMs resonators potentially have very high Qs in the MIR/FIR, this does not translate into very long effective path length for chemical sensing, especially if we try to sense gas phase chemicals. This is because the evanescent wave only accounts for a small portion of the Electro Magnetic (EM) field of the WGMs, and only the evanescent wave interacts with external chemicals. The fraction of the WGM's energy in the evanescent field depends on the refractive indices inside and outside resonator. For gas phase sensing, this fraction, $f$, is only $\sim 1 \%$ or less[23]; while for liquid phase, $f$ could be as high as $30 \%[22]$. Therefore, with the $\mathrm{Q}$ value of $>10^{8}$ in the gas phase and $>10^{7}$ in the liquid phase, the effective sensor path length would exceed 1 meter. One meter path length would be sufficient for liquid sensing but in the gas phase it is much shorter of the path lengths provided by multipass cells (1 >100 meters) and cavity enhanced methods (>1,000meters). However, WGM sensors could be used in applications that require small sample volume or overall small size of the sensors. For example, they could be used as inline sensor for Gas Chromatography (GC). With a one meter path length one could detect ppbV concentrations of $\mathrm{CO}_{2}$, which would exceed the sensitivity of the mainstream GC sensors.

\section{CONCLUSION}

We analyzed the thermal induced frequency tuning mechanisms in QC lasers, and report the improved modulation and modification scheme for improving the tuning range and speed of QC lasers. The applications of widely tunable single frequency laser, i.e. measuring chemicals in water, and coupling with the hollow waveguide and Whispering Gallery Mode cavity and use as sensors for chemicals are demonstrated and discussed.

We gratefully acknowledge the helpful discussions on WGMs with Dr. Ivan Grudinin, Dr. Andrey Matsko and Dr. Vladmir Ilchenko, and also on DFB QC lasers with Dr. Jenyu Fan and Dr. Xiaojun Wang of Adtech Optics.

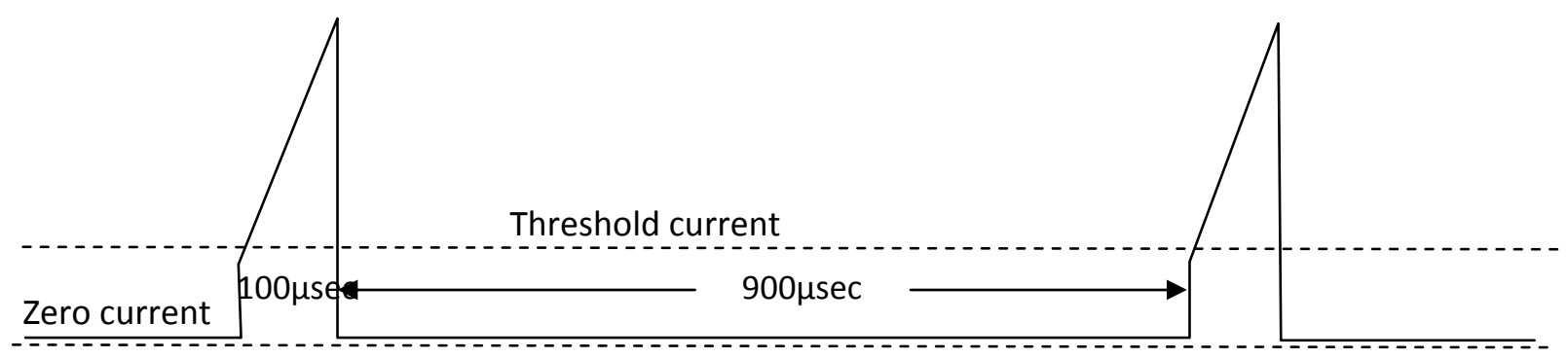

Figure 1. Low duty cycle wide frequency tuning current ramping scheme. The low duty cycle will give the lowest initial AR temperature, and fast pulsing will realize $200 \mathrm{~K}$ localized temperature gradient. 


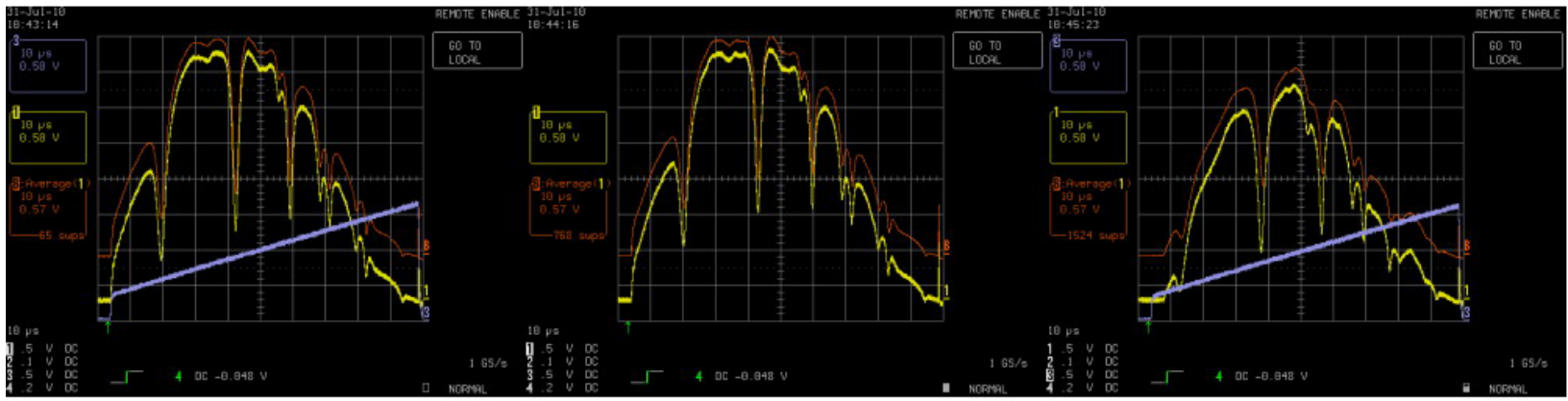

-25C_2.1M_1

-15C_2.1M_1

-7C_2.1M_1

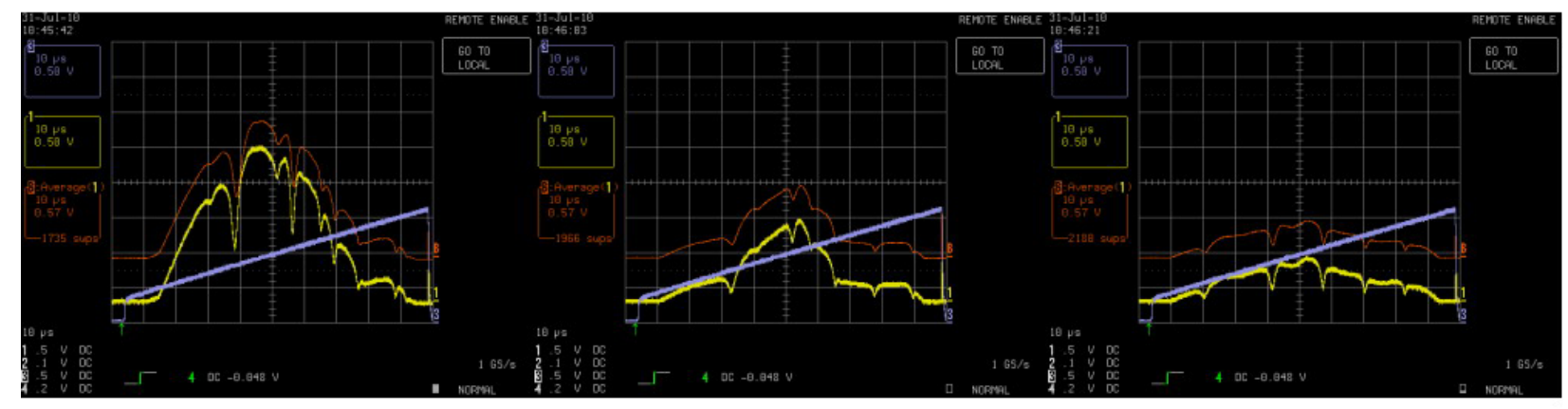

-4C_2.1M_1

$-0 \mathrm{C} \_2.1 \mathrm{M} \_1$

6C_2.1M_1

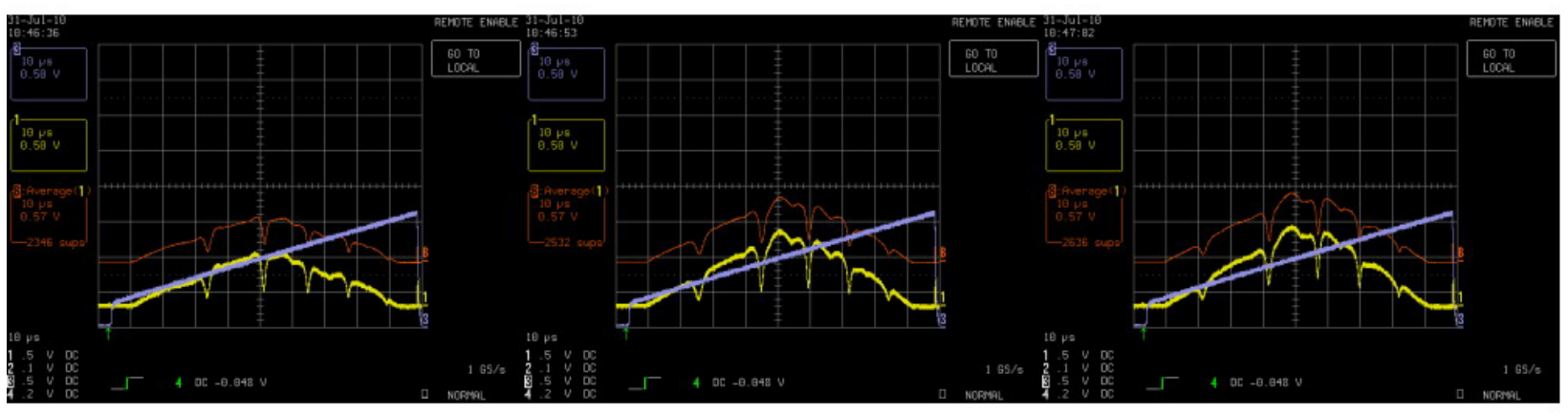

Proc. of SPIE Vol. 7945 794506-9 


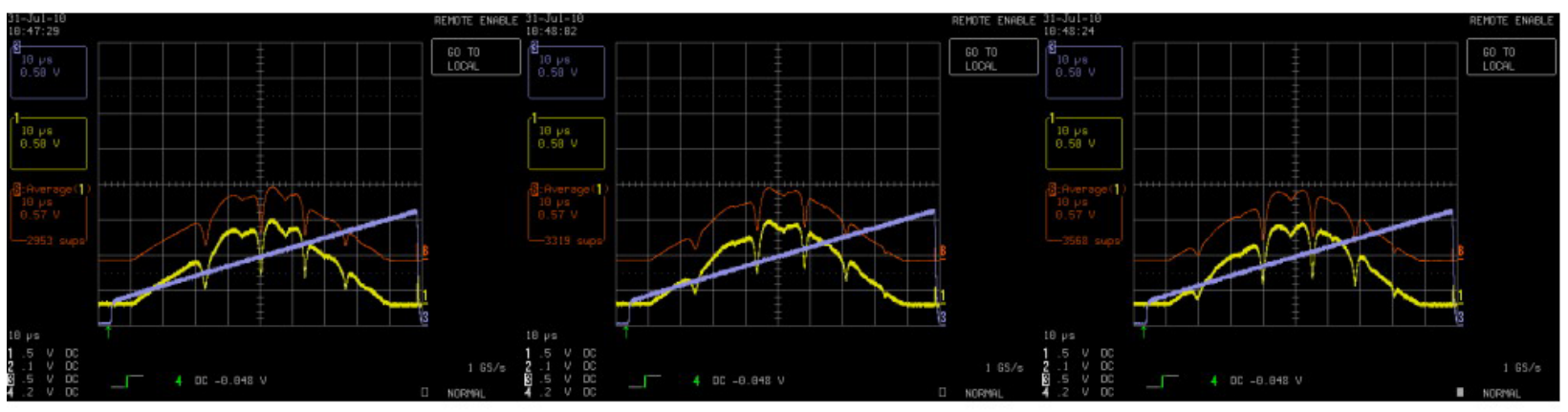

$20 C_{-} 2.1 M_{-} 1$

22C_2.1M_1

$25.7 C_{-} 2.1 \mathrm{M} \_1$

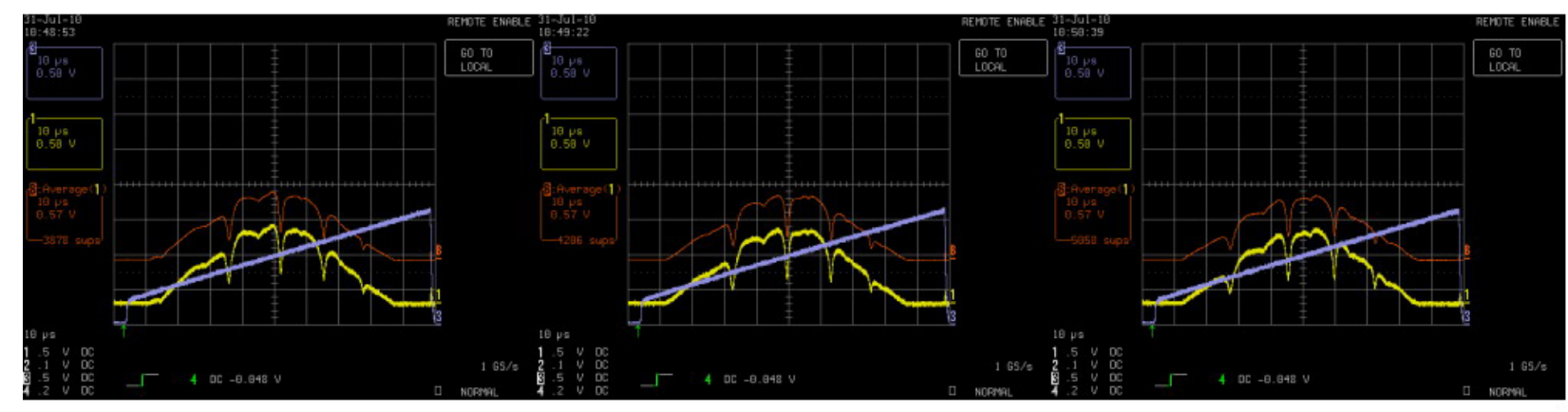

28.1C_2.1M_1

28.6C_2.1M_1

$-17 C_{-} 2.1 M_{-} 2$

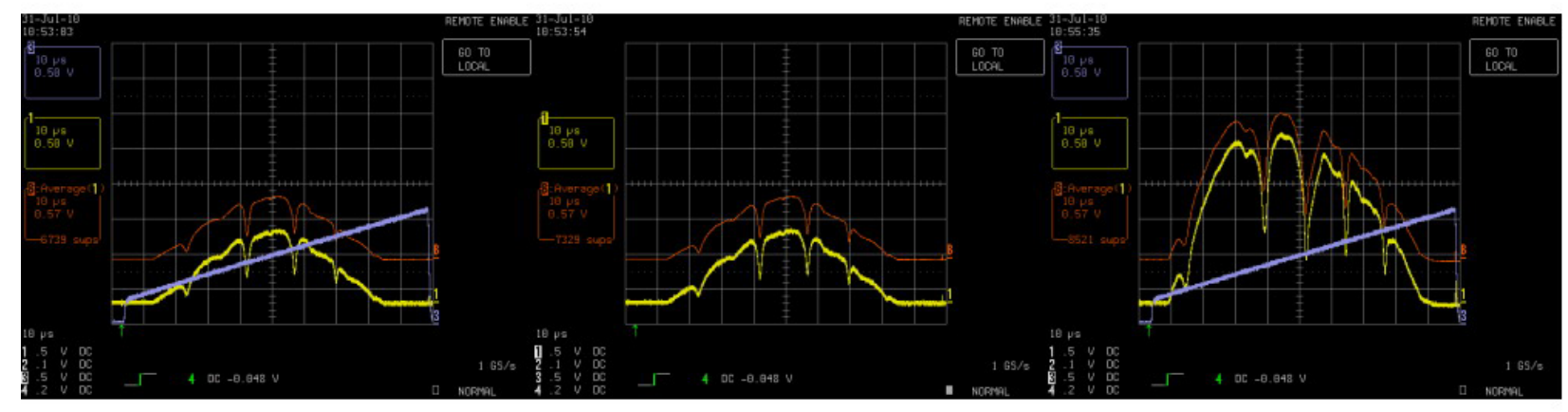

Figure 2 . With $2 \%$ duty cycle pulse and $100 \mu$ sec pulse width $(200 \mathrm{~Hz})$, as TEC temperature change from $+28^{\circ} \mathrm{C}$ to $-45^{\circ} \mathrm{C}$, the wavelength scan range changed from 4 peaks to 6 peaks of ${ }^{13} \mathrm{CO} 2$, corresponding to $\sim 9 \mathrm{~cm}^{-1}$ to $15 \mathrm{~cm}^{-1}$. Also, the power goes up 3 times, while the effective current tuning range changed increased 7 units to 10 . 


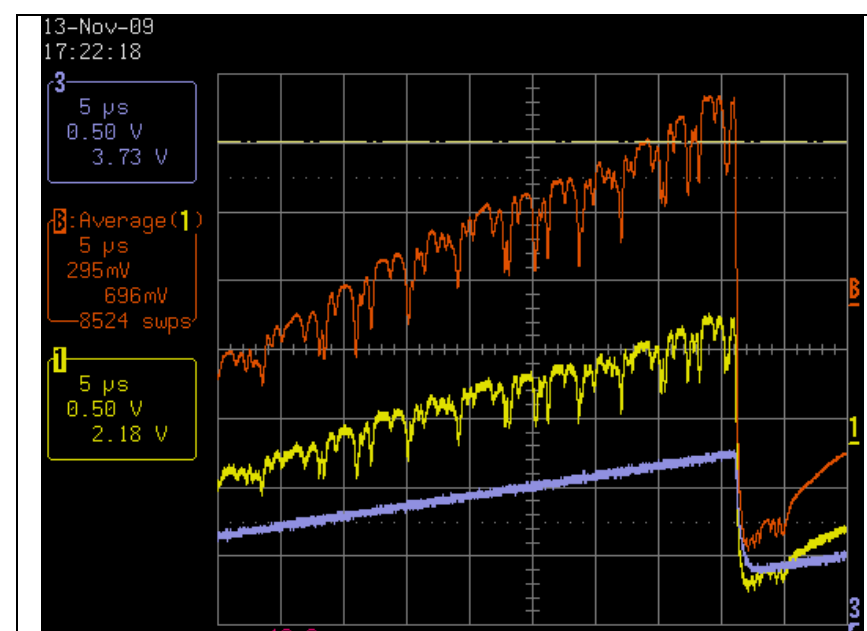

Fig. 3a. Coupling angle smaller than critical angle from $\mathrm{ZnSe}$ to

$\mathrm{CaF}_{2}$

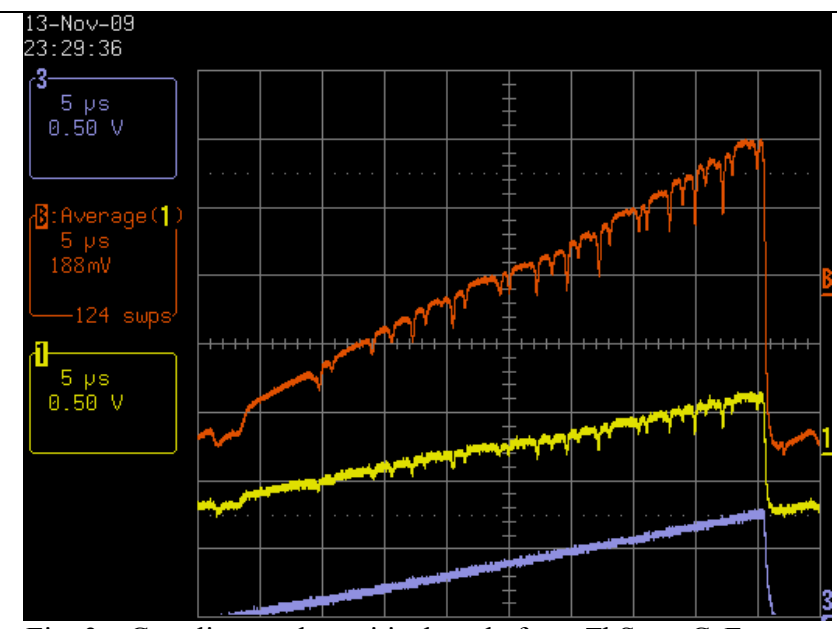

Fig. 3c. Coupling angle $\sim$ critical angle from $\mathrm{ZbSe}$ to $\mathrm{CaF}_{2}$, resulting much reduced simplified spectra

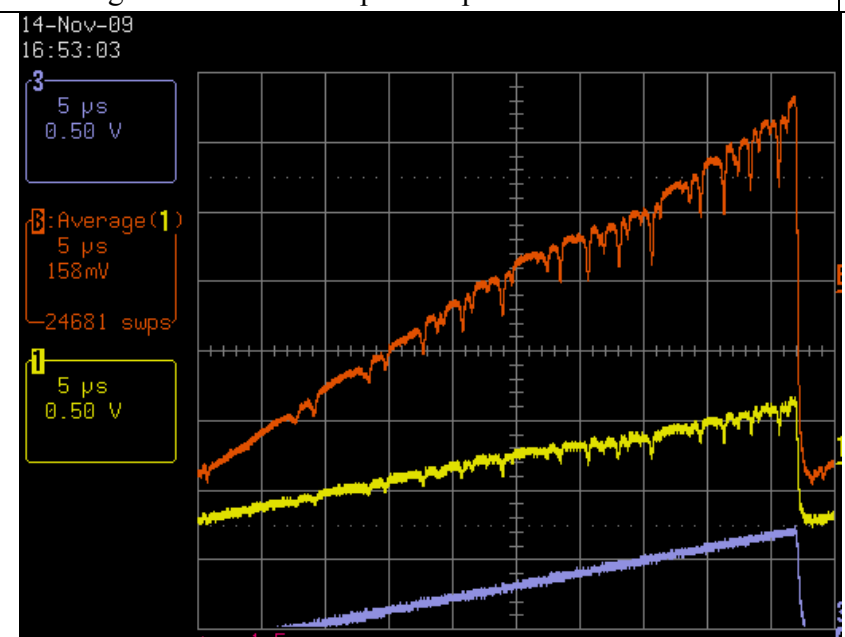

Fig. 3e. WGM spectra with period about $75 \mu \mathrm{sec}$ or $0.47 \mathrm{~cm}^{-1}$

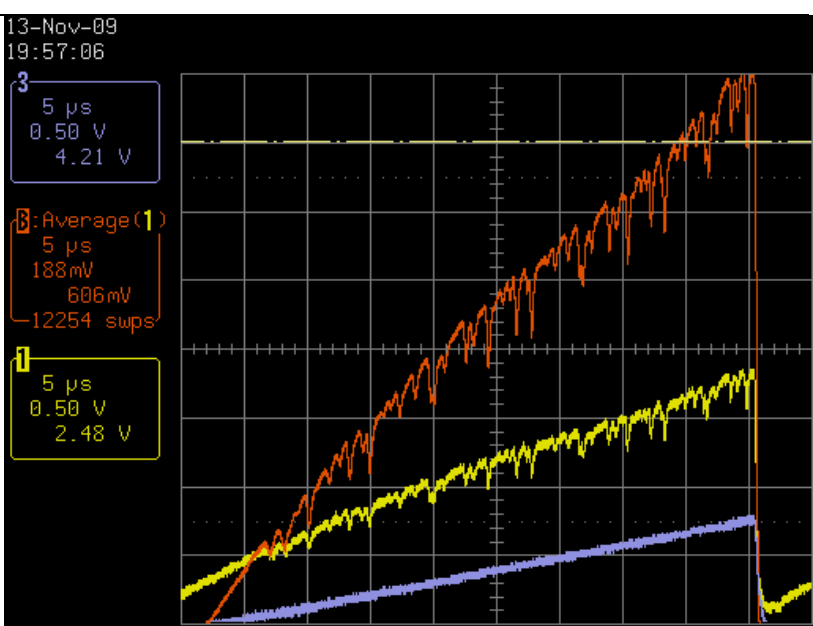

Fig. 3b. Coupling angle closer to critical angle from $\mathrm{ZnSe}$ to $\mathrm{CaF} 2$

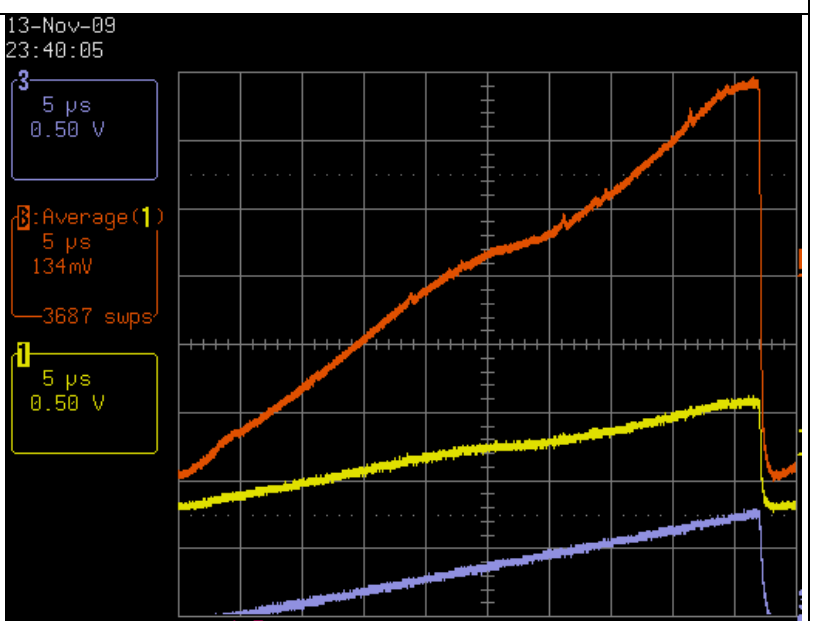

Fig. 3d. Coupling angle $>$ critical angle from $\mathrm{ZS}$ to $\mathrm{CF}$, only reversed spectra are observed

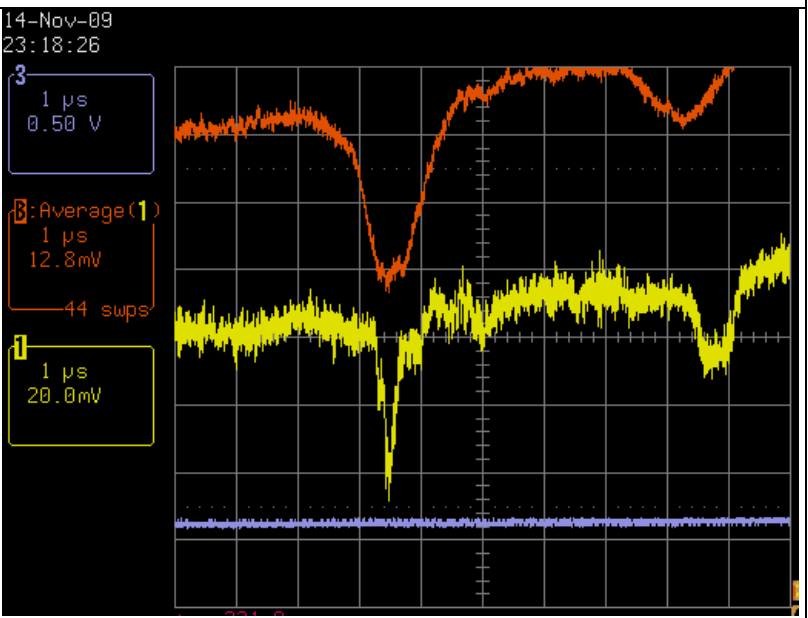

Fig. 3f. Zoom-in single scan (below) FWHM of $<0.2 \mu \mathrm{Sec}$, or $\sim 0.001 \mathrm{~cm}^{-1}$. The Q should be larger than $2 \times 10^{6}$ at $2,280 \mathrm{~cm}^{-1}$. 

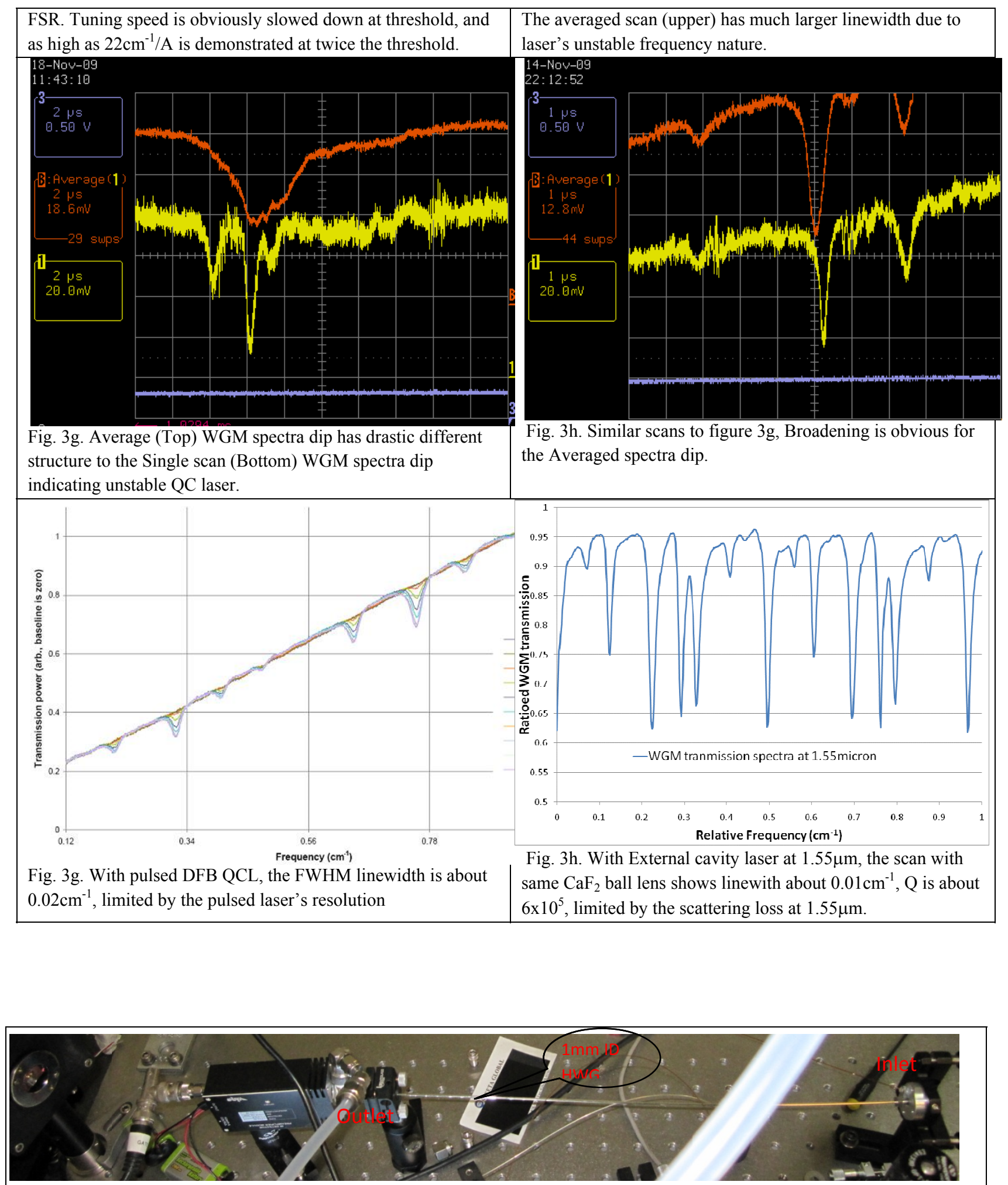

Figure 4a on top shows the setup of the HWG and QCL spectrosmeter under low vacuum conditions. 


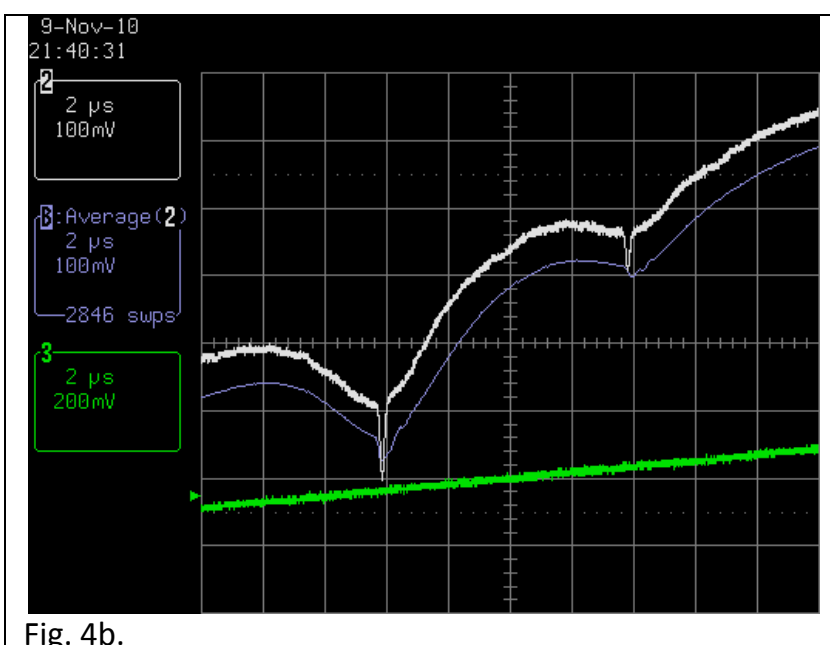

Fig. 4b.

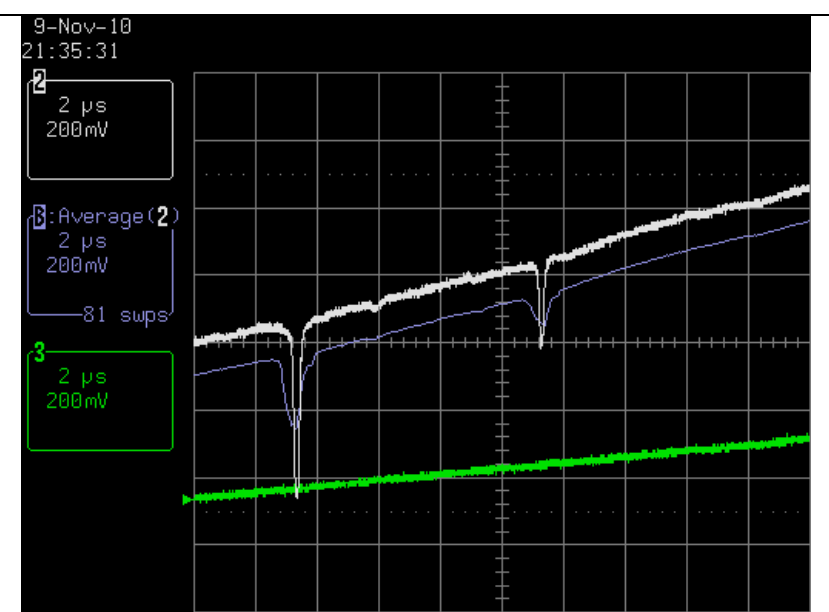

Fig. 4c.

Figure $4 \mathrm{~b}$ on the middle left shows the spectra taken when the DFB QC laser is launched into the HWG after passing a fairly long open path, during which the air CO2 absorption created a pair of pressure broadened lines, and then a pair of narrow $\mathrm{CO} 2$ lines show up as a result of Doppler free absorption inside the low vacuumed HWG. Figure $4 \mathrm{c}$ on the middle right shows the spectra when the DFB QC laser is launched immediately into the HWG after passing only 1 " of open path where the broadened absorption is not visible. The green line is the ramping current; the white line is the single scan absorption signal; the blue line is the results after average 15 scans. The figure on the bottom right shows that at higher concentration of

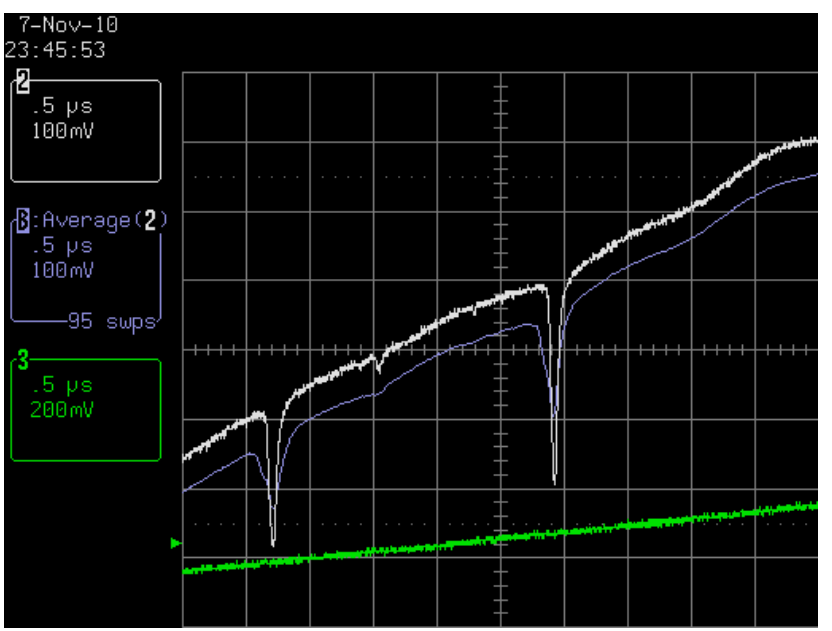
$\mathrm{CO} 2$, there are minor lines of other $\mathrm{CO} 2$ absorptions which will interfere with measurement of the main lines if the linewidth is limited to $0.1 \mathrm{~cm}^{-1}$.

\section{REFERENCES}

[1] Griffiths, P.R., J.A. Dehaseth, and L.V. Azarraga, "Capillary Gc/Ft-Ir" Analytical Chemistry, 55(13), 1361-\&.(1983)

[2] Wu, S., et al., "Hollow waveguide quantum cascade laser spectrometer as an online microliter sensor for gas chromatography" Journal of Chromatography A, 1188(2), 327-330 (2008)

[3] Lyakh, A., et al., "3 W continuous-wave room temperature single-facet emission from quantum cascade lasers based on nonresonant extraction design approach" Applied Physics Letters, 95(14),(2009).

[4] Razeghi, M., et al., "High power quantum cascade lasers," New Journal of Physics, 11, (2009).

[5] Hugi, A., R. Maulini, and J. Faist, "External cavity quantum cascade laser" Semiconductor Science and Technology, 25(8), p. 14 (2010).

[6] Guo, B.J., et al., "Mid-infrared laser measurements of aqueous glucose," Journal of Biomedical Optics, 12(2), (2007). 
[7] Guo, B.J., et al., "Laser-based mid-infrared reflectance imaging of biological tissues," Optics Express, 12(1), 208-219 (2004).

[8] Schaden, S., et al., "Direct determination of carbon dioxide in aqueous solution using mid-infrared quantum cascade lasers," Applied Spectroscopy, 58(6), 667-670 (2004).

[9] Ye, J., et al., "Highly selective terahertz optical frequency comb generator," Optics Letters, 22(10), 746-746 (1997).

[10] Kelly, B., et al., "Discrete mode laser diodes with very narrow linewidth emission," Electronics Letters, 43(23), 1282-1284 (2007).

[11] A. Yariv, "Quantum Electronics," third ed., Wiley, New York, p. 577-597 (1989).

[12] Myers, T.L., et al., "Free-running frequency stability of mid-infrared quantum cascade lasers," Optics Letters, 27(3), 170-172 (2002).

[13] Taubman, M.S., et al., "Frequency stabilization of quantum-cascade lasers by use of optical cavities," Optics Letters, 27(24), 2164-2166 (2002).

[14] Mukherjee, N. and C.K.N. Patel, "Molecular fine structure and transition dipole moment of NO2 using an external cavity quantum cascade laser," Chemical Physics Letters, 462(1-3), 10-13 (2008). [15] Borri, S., et al., "Lamb-dip-locked quantum cascade laser for comb-referenced IR absolute frequency measurements," Opt. Express, 16(15), 11637-11646 (2008).

[16] Letokov, V.S., "Saturation Spectroscopy" K. Shimoda (Ed.) High Resolution Laser Spectroscopy, Springer., 96-171 (1976).

[17] Radloff, W., "Gas-Analysis by Non-Linear Absorption Spectroscopy,". Kvantovaya Elektronika,5(11), 2358-2368 (1978).

[18] Dilorenzo, O., P.C. Deoliveira, and J.R.R. Leite, "Saturation Spectroscopy of Infrared MolecularTransition in an Optically Thick Cell," Optics Letters, 16(22), 1768-1770 (1991).

[19] Radloff, W. and H.H. Ritze, "Nonlinear Absorption Spectroscopy of Ethane Molecule," Applied Physics, 14(4), 343-350 (1977).

[20] Matsko, A.B. and V.S. Ilchenko, "Optical resonators with whispering-gallery modes - Part I: Basics," leee Journal of Selected Topics in Quantum Electronics, 12(1), 3-14 (2006).

[21] Ilchenko, V.S. and A.B. Matsko, "Optical resonators with whispering-gallery modes - Part II: Applications," leee Journal of Selected Topics in Quantum Electronics, 12(1), 15-32(2006).

[22] Armani, A.M. and K.J. Vahala, "Heavy water detection using ultra-high-Q microcavities," Optics Letters, 31(12), 1896-1898 (2006).

[23] Farca, G., S.I. Shopova, and A.T. Rosenberger, "Cavity-enhanced laser absorption spectroscopy using microresonator whispering-gallery modes," Optics Express, 15(25), 17443-17448 (2007).

[24] Grudinin, I.S. and L. Maleki, "Efficient Raman laser based on a CaF2 resonator," Journal of the Optical Society of America B-Optical Physics, 25(4), 594-598 (2008).

[25] Wittmann, A., et al., "'Distributed-Feedback Quantum-Cascade Lasers at 9 mu m Operating in Continuous Wave Up to 423 K," leee Photonics Technology Letters, 21(9-12), 814-816 (2009).

[26] Coldren, L. and S. Corzine, "Laser diode and Photonic Integrated Circuits," Wiley-Interscience Publication (1995) 
[27] Diehl, L., et al., "High-power quantum cascade lasers grown by low-pressure metal organic vaporphase epitaxy operating in continuous wave above 400 K," Applied Physics Letters, 88(20), (2006).

[28] Grudinin, I.S., et al., "Ultra high Q crystalline microcavities," Optics Communications, 265(1), 3338.(2006).

[29] Duxbury, G., et al., "Quantum cascade laser spectroscopy: diagnostics to non-linear optics" Journal of Modern Optics, 56(18-19), 2034-2048 (2009) 